\title{
Subsampled Factor, Passband Frequency Range and Filter Order Used For Design of Oversampled Filter Bank for 2d Image Analysis
}

\author{
${ }^{1}$ Dr.Mrs.S.R.Chougule, ${ }^{2}$ Dr.Smt.R.S.Patil \\ ${ }^{I}$ Professor and Principal BharatiVidyapeeth's C.O.E.Kolhapur \\ ${ }^{2}$ Professor and Principal, Dr.D.Y.Patil C.O.E.Talasande
}

\begin{abstract}
This paper proposes an oversampled filter bank, which gives the resultant near perfect reconstruction of input image. An oversampled filter bank structure that can be implemented using popular and efficient fast filter banks to allow subband processing of an input signal with substantially reduced aliasing between subbands. For filters design direct form-II structure FIR filters are used and transformed into two dimensional forms using frequency transformation techniques. Investigation of oversampled filter bank using different oversampled factors and different filter orders for different images are carried out. The analysis of outputs of filter banks images are carried out by using peak signal to noise ratio [PSNR] of compressed images. By using local thresholding segmentation where major components of images are exposed and analysis of filter bank output images are carried out which is helpful to find the difference between images.
\end{abstract}

\section{Introduction:}

Oversampled filter banks have found use in a variety of applications in recent years. In particular, they have found commercial applications in low-power audio signal processing for devices such as hearing aids [1]. Other researchers have also highlighted their potential in audio processing for applications such as acoustic echo cancelation, dynamic range compression and noise reduction. For audio processing devices such as hearing aids, highly oversampled filter banks offer a compromise between aliasing reduction in each subband and achieving ultra low delay through the filter bank. Subband decomposition and coding of images have become quite popular in the last two decades. Subband coding has been applied to speech signals. While most of the research in the area of subband decomposition concentrated on 1D signal and on separable approaches for multidimensional signal. Daubechies [2] and Mallat [3] have developed the theory of wavelets and have shown that subband coding and wavelets are closely related. In subband signal coding, the basic objective is to concentrate the signal energy in as few subspectra or subbands as possible for efficient transmission of information. The uneven distribution of signal energy over the frequency band provides the basis for source compression techniques, thus data compression is the driving motivation for subband signal coding. In subband coding, the frequency band of the signal is first divided into a set of uncorrelated frequency bands by filtering and then each of these subbands is encoded.[4]. Multirate filter banks find applications in subband decomposition systems. The complete filter bank is composed of two sections: the analysis section which decomposes the signal into a set of subband components and the synthesis section which reconstructs the signal from its components.

The subband analysis and synthesis filters should be designed to be alias-free and should satisfy the perfect signal reconstruction property. The simultaneous cancellation of aliasing as well as amplitude and phase distortions leads to perfect reconstruction (PR) filter banks which are suitable for hierarchical subband coding and multiresolution signal decomposition. This paper presents a simplified method for designing prototype FIR filter designs. This approach will extend similar approaches used in conventional critically sampled near perfect reconstruction [NPR] filter banks. The goal of this design method is to develop a simple and flexible method that can be applied for a wide variety of oversampled filter bank configure rations. Design of filters of filter bank is major point in the filter bank. Here direct form-II filter structures are used for filters of filter bank. This paper investigate design of proper oversampled filter bank which shows the resultant near perfect reconstruction of input. Similarly different subsampled factors are used for analysis of different images. Section 3 gives the fifteen channel oversampled FIR filter bank. Section 3 highlights design method of oversampled FIR filter bank. Section 4 shows discussion with results. 
II. M-Channel Multirate Filter Bank :

The generalized M-channel multirate filter bank has the form shown in Figure. 1.

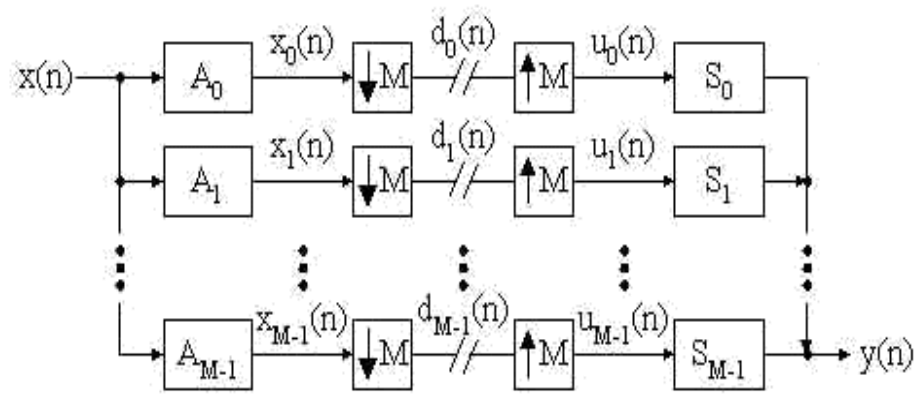

Figure. 1. A generalized M-channel multirate filter bank.

The output of this system can be expressed as

$$
\mathrm{Y}(\mathrm{z}) \sum_{\mathrm{p}=0}^{\mathrm{M}-1} \mathrm{G}_{\mathrm{p}}(\mathrm{z}) \mathrm{X}\left(\mathrm{z} \mathrm{w}_{\mathrm{p}}\right)
$$

where

$$
G_{p}(z)=\frac{1}{M} \sum_{k=0}^{M-1} A_{k}(z W y) \cdot S_{k}(z), \quad 0 \leq p \leq M-1
$$

and $\mathrm{W}$ represents the phase term, $\mathrm{e}^{-\mathrm{j} 2 \square / \mathrm{M}}$ [7]. The alias terms are readily identified as $\mathrm{X}\left(\mathrm{z} . \mathrm{W}^{\mathrm{p}}\right)$ since they represent identical, but frequency shifted versions of $\mathrm{X}(\mathrm{z})$. $\mathrm{G}_{\mathrm{p}}(\mathrm{z})$, then, is the associated gain factor for a given alias term. Thus, the filter bank is free from aliasing only if

$$
\mathrm{G}_{\mathrm{p}}(\mathrm{z})=0 \quad \text { for } \quad 1 \leq \mathrm{p} \leq \mathrm{M}-1
$$

Once again, this alias free system can be represented as a single transfer function of slightly different form than that of the two-channel QMF case.

$$
Y(z)=G_{0}(z) X(z)=\left(\frac{1}{M} \sum_{k=0}^{M-1} A_{k}(z) S_{k}(z)\right)^{\prime}(z)
$$

The criteria for amplitude and phase distortion are the same. If $\mathrm{G}_{0}(\mathrm{z})$ is not allpass, the filter bank suffers from amplitude distortion. If $\mathrm{G}_{0}(\mathrm{z})$ does not have linear phase, the filter bank suffers from phase distortion. Again, when the filter bank is free from aliasing, amplitude distortion, and phase distortion, it is called a PR filter bank. M-channel banks lend themselves to matrix representations which are useful in the design of specific filters. Figure. $2 \mathrm{a}$ and $\mathrm{b}$ illustrate the analysis and synthesis filter matrix equations in polyphase form . Figure. 2c illustrates the new M-channel filter bank after simplifying with the noble identities. Notice the two new matrix equations this representation yields.

$$
A(z)=\mathbf{E}\left(z^{M}\right) \mathbf{d}(z)
$$

and

$$
S^{T}(z)=z^{M-1} \cdot d^{T}\left(z^{1}\right) \mathbf{R}\left(z^{M}\right)
$$

where $\mathrm{A}$ and $\mathrm{S}$ represent the analysis and synthesis matrices, respectively, and $\mathrm{d}$ represents the delay chain vector. The bulk of the material covered in this paper will concentrate on the specific case where $M=2$. Figure 3 illustrate the associated polyphase matrices for this case. 


$\left[\begin{array}{c}A_{0}(z) \\ A_{1}(z) \\ \vdots \\ A_{M-1}(z)\end{array}\right]=\left[\begin{array}{cccc}E_{00}\left(z^{M}\right) & E_{01}\left(z^{M}\right) & \cdots & E_{0, M-1}\left(z^{M}\right) \\ E_{10}\left(z^{M}\right) & E_{11}\left(z^{M}\right) & \cdots & E_{1, M-1}\left(z^{M}\right) \\ \vdots & \vdots & \cdots & \\ E_{M-1,0}\left(z^{M}\right) & E_{M-1,1}\left(z^{M}\right) & \cdots & E_{M-1, M-1}\left(z^{M}\right)\end{array}\right]\left[\begin{array}{c}1 \\ z^{-1} \\ \vdots \\ z^{(M-1)}\end{array}\right]$

(a)

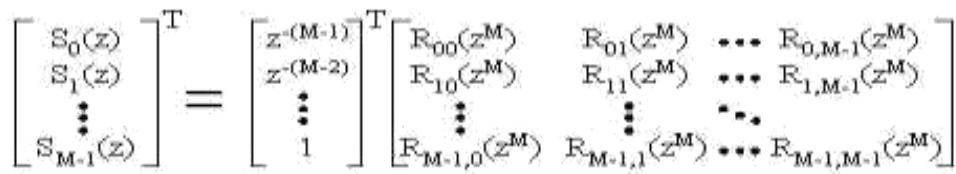

(b)

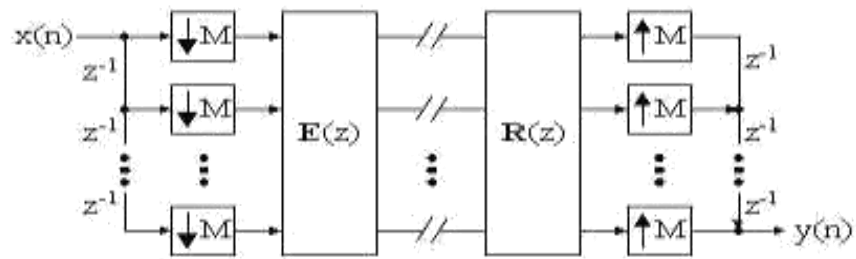

(c)

Figure 2. The polyphase representation for an M-channel (a) analysis bank, $A(z)=E\left(z^{\mathrm{M}}\right) \mathrm{d}(\mathrm{z})$, and (b) synthesis bank, $S^{T}(z)=z^{-(M-1)} d^{T}\left(z^{-1}\right) R\left(z^{M}\right)$. (c) The M-channel filter bank after simplifying with the noble identities.

It can be shown that the system illustrated in Figure. $2 c$ exhibits perfect reconstruction if

$$
\mathbf{R}(\mathrm{z}) \mathbf{E}(\mathrm{z})=\mathbf{c z} \mathbf{I}
$$

or, more generally, if

$$
\begin{aligned}
& \mathbf{R}(z) \mathbf{E}(z)=\mathbf{c z}^{-\mathrm{m}}\left[\begin{array}{cc}
\mathbf{0} & \mathbf{I}_{\mathrm{M}-\mathrm{t}} \\
\mathbf{z}^{-1} \mathbf{I}_{1} & \mathbf{0}
\end{array}\right] \\
& {\left[\begin{array}{l}
A_{0}(z) \\
A_{1}(z)
\end{array}\right]=\left[\begin{array}{ll}
E_{00}\left(z^{2}\right) & E_{01}\left(z^{2}\right) \\
E_{10}\left(z^{2}\right) & E_{11}\left(z^{2}\right)
\end{array}\right]\left[\begin{array}{c}
1 \\
z-x
\end{array}\right]} \\
& {\left[\begin{array}{l}
s_{0}(z) \\
s_{1}(z)
\end{array}\right]^{T}=\left[\begin{array}{c}
z^{-1} \\
1
\end{array}\right]^{T}\left[\begin{array}{ll}
R_{00}\left(z^{2}\right) & R_{01}\left(z^{2}\right) \\
R_{10}\left(z^{2}\right) & R_{11}\left(z^{2}\right)
\end{array}\right]}
\end{aligned}
$$

(b)

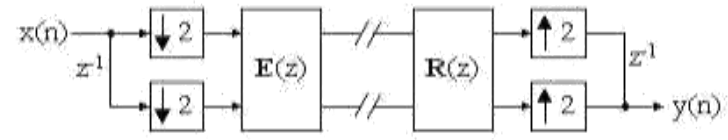

(c)

Figure 3. The polyphase matrix representation of a two-channel (a) analysis bank and (b) synthesis bank and (c) the polyphase system after simplification.

for some integer, $\mathrm{r}$, between 0 and $\mathrm{M}-1$, some integer, $\mathrm{m}$, and some non-zero constant, $\mathrm{c}$. If one of these conditions holds, $y(n)=c x\left(n-n_{0}\right)$ where $n_{0}=M m+r+M-1$ regardless of whether the system is FIR or IIR. Subsequently, if Equation 8 is satisfied,

$$
\text { det } \mathbf{R}(z) \text { det } \mathbf{E}(z)=\mathbf{c} \mathbf{z}^{\mathrm{k}}
$$

for some constant, c, and some integer, $\mathrm{k}$. If the analysis and synthesis filters are FIR, then their coefficient matrices and determinants are FIR. Thus, every FIR PR system must then satisfy

$$
\operatorname{det} \mathbf{E}(z)=\mathrm{az}^{\mathrm{kO}} \text { and } \quad \operatorname{det} \mathbf{R}(\mathrm{z})=\mathrm{bz}
$$


for constant, a, b, k0, and k1. By using above design steps filter bank for 14 channels are selected and applied for $2 \mathrm{D}$ images(i.e. tif and gif images). The analysis and synthesis filters are designed by using above technique. Filters of filter bank implemented in one dimensional form and then transformed into 2D form for 2D image application. Downsampling and upsampling of image is carried out by using bilinear interpolation technique. Clearly, the concept of multirate filtering relies on the two processes that effectively alter the sampling rate, decimation and expansion. Decimation or downsampling by a factor of $\mathrm{M}$ essentially means retaining every $\mathrm{M}^{\text {th }}$ sample of a given sequence. Decimation by a factor of $\mathrm{M}$ can be mathematically defined as

$$
\mathrm{x}(\mathrm{n})_{\mathrm{hM}}=\mathrm{x}(\mathrm{M} \cdot \mathrm{n})
$$

or equivalently,

$$
\mathrm{X}(\mathrm{z})_{\prod_{\mathrm{M}}}=\frac{1}{\mathrm{M}} \sum_{\mathrm{m}=0}^{\mathrm{M}-1} \mathrm{X}\left(\mathrm{W}_{\mathrm{M}}^{\mathrm{m}} \mathrm{z}^{\mathrm{Im}}\right) \quad \text { where } \quad \mathrm{W}^{\mathrm{M}}=\mathrm{e}^{-12 \pi \mathrm{M}}
$$

Expansion or upsampling by a factor of $\mathrm{M}$ essentially means inserting $\mathrm{M}-1$ zeros between each sample of a given sequence. Expansion by a factor of $\mathrm{M}$ can be mathematically defined as

$$
\mathrm{x}(\mathrm{n})_{\mathrm{M}}= \begin{cases}x(\mathrm{n} / \mathrm{M}) & \text { for integer } \mathrm{nM} \\ 0 & \text { oflerwise }\end{cases}
$$

or equivalently,

$$
\mathrm{X}(\mathrm{z})_{\mathbb{M}}=\mathrm{X}\left(\mathrm{z}^{\mathrm{M}}\right)
$$

Downsample algorithm reduces the size of image by downsample factor and upsample algorithm restores original size of image.

\section{Oversampled filter bank:}

Figure 1 shows a general filter bank structure comprising an analysis and a synthesis stage. The analysis filter bank splits a fullband signal $\mathrm{X}(\mathrm{z})$ into $\mathrm{N}$ frequency bands by a series of bandpass filters $\mathrm{Hk}(\mathrm{z}), \mathrm{k}$ $=0, \ldots,(\mathrm{N}-1)$ and decimates by a factor $\mathrm{M} \leq \mathrm{N}$, resulting in subband signal. The dual operation of reconstructing a fullband signal from the $\mathrm{N}$ subband signals is accomplished by a synthesis filter bank, where upsampling by $M$ is followed by interpolation filters $F_{k}(z)$. The purpose of oversampling by a ratio N/M > 1 rather than a critical decimation. Filter bank comprises of a series of bandpass filters. Non critical decimation of the resulting subbands will permit the benefit of lower computational complexity and avoiding aliasing in the subbands by selecting proper frequency bands with proper oversample factor $\mathrm{M}$.

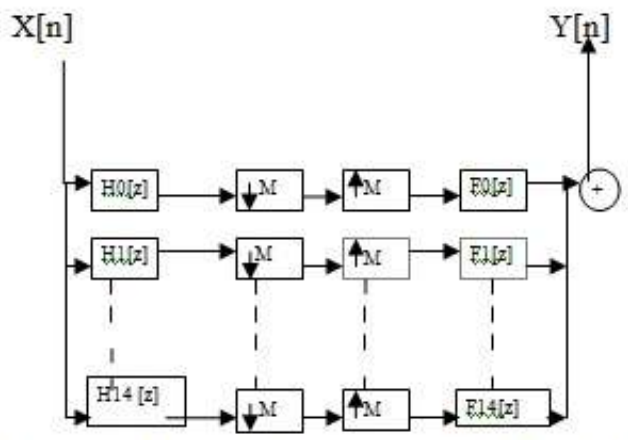

Figure 4: Fifteen channel oversampled filter bank

Oversampled filter bank is designed by selecting different filter orders and different oversample factor. This is helpful to investigate proper oversample filter bank which shows resultant near perfect reconstruction of input signal. This design is suitable for even filter order filters. Direct form II transposed structure FIR filters are used for filter banks. Here selection of frequency bands are important which shown in figureure5. As per as the filter order changes there is change in side lobes of filters, and result in variation of output signal. Similarly this filter bank is designed with different oversampling factors[5,6], which is helpful to analyze proper oversample factor to achieve resultant near perfect reconstruction of input image. The analysis of two dimensional image is carried out by using above oversampled filter bank. At analysis bank the size of filtered image is reduced by downsample factor, means rows and columns of image reduces. Similarly at the synthesis bank stage the original size of image is restored by using same upsample factor, means the rows and columns of 
image upsampled and applied for synthesis filters. Selection of filters of synthesis bank is same as analysis bank. Finally filtered output of synthesis bank is added and output image is analyzed .

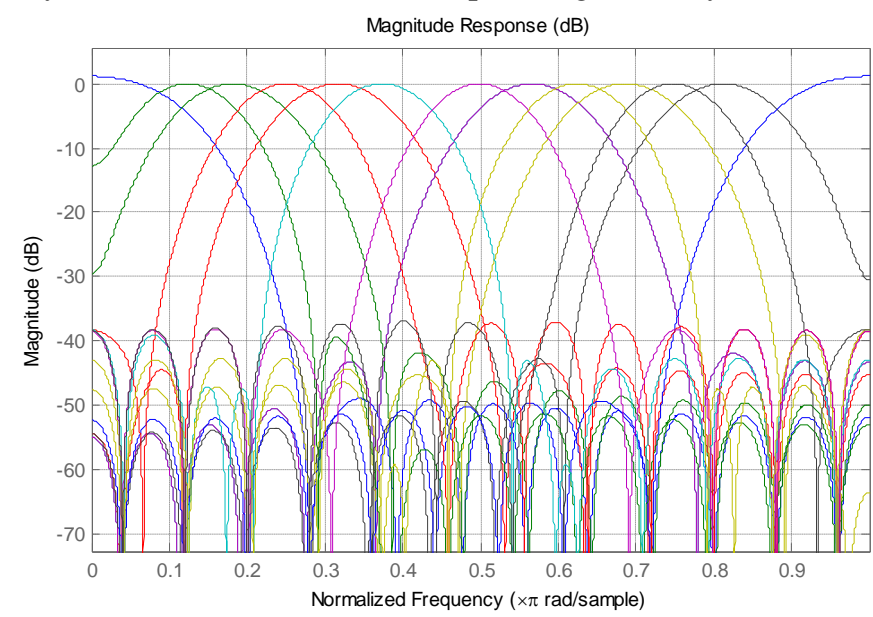

Figure 5: Normalized frequency response of 15 channel

\section{Experimental Results and Discussion:}

Initially design of proposed oversampled FIR filter bank using direct form II transposed structure FIR filters are developed in one dimensional (1D) form. Band pass filters are used for design of filter bank channels .1D filter transformed into two dimensional (2D) using frequency transformation techniques, which is discussed in section III. Initially input signal is divided into 8 channels but at transition gap some frequency components are lost means not passed for further processing [5,6]. Therefore to overcome this problem extra seven filters are selected at the spectral gaps and all the frequency components are passed at output stage. Figureure1 shows fifteen channel oversampled FIR filter bank. The normalized frequency spectrum of 15 channel filter bank with filter order 28 is as shown in figure 5 . Selection of frequency bands of band pass filters are important in this proposed filter bank. Synthesis filters are designed same like analysis filters of filter bank for each channel. Design of downsampler and upsampler of analysis and synthesis filter bank is carried out by using bilinear interpolation technique. In downsampler block of each channel size of image with rows and columns are reduced by downsample factor. Similarly at the upsampler block, image size is restored by selecting same upsampler factor. Initially downsample and upsample factor 8 is selected and resultant of fifteen channel oversampled filter bank with different filter order with DCT compression is as shown in figureure4a,b,c .Similarly same filter bank with oversample factor 4 and different filter order is developed and resultant is as shown in figure 7d,e,f Finally filter bank is designed with downsample factor 2 with different filter order and investigation of oversampled filter bank near perfect reconstruction input is carried out. This filter bank is applied for different images namely Barba,Camerman and man in tif format and Lena image with gif format . Here for filter bank, investigation of oversample factor with proper filter order is carried out, which shows resultant near perfect input. Resultant of Barba image for different filter order and oversample factor 2 with DCT compression is as shown in figure. $8 \mathrm{i}$ to $8 \mathrm{p}$.

Proposed oversampled filter banks results are applied for image compression. Out of the image compression techniques available, transform coding is the preferred method. Since energy distribution varies with each image, compression in the spatial domain is not an easy task. Images do however tend to compact their energy in the frequency domain making compression in the frequency domain much more effective. Transform coding is simply the compression of the images in the frequency domain. Transform coefficients are used to maximize compression. For lossless compression, the coefficients must not allow for the loss of any information. The DCT is fast. It can be quickly calculated and is best for images with smooth edges like photos with human subjects. The DCT coefficients are all real numbers unlike the Fourier Transform. The Inverse Discrete Cosine Transform (IDCT) can be used to retrieve the image from its transform representation.

DCT:

$\frac{C(v)}{2} \frac{C(u)}{2} \sum_{y=0}^{7} \sum_{x=0}^{7} s(y, x) \cos [(2 x+1) k \pi / 16] \cos [(2 y+1) v \pi / 16]$

IDCT:

$\sum_{v=0}^{7} \frac{C(v)}{2} \sum_{u=0}^{7} \frac{C(u)}{2} S(v, u) \cos [(2 x+1) u \pi / 16] \cos [(2 y+1) v \pi / 16]$ 
After image compression all the results are applied for peak signal to noise ratio [PSNR] estimation. The PSNR is most commonly used as a measure of quality of reconstruction in image compression. It is most easily defined via the mean squared error (MSE) which for two $m \times n$ monochrome images $I$ and $K$ where one of the images is considered a noisy approximation of the other is defined as:

$$
M S E=\frac{1}{m n} \sum_{i=0}^{m-1} \sum_{j=0}^{n-1}\|I(i, j)-K(i, j)\|^{2} \quad \text { The PSNR is defined as: }
$$

Here, $M A X_{I}$ is the maximum pixel value of the

$$
P S N R=10 \cdot \log _{10}\left(\frac{M A X_{I}^{2}}{M S E}\right)=20 \cdot \log _{10}\left(\frac{M A X_{I}}{\sqrt{M S E}}\right)
$$

image. Comparison of all the results of filter banks in terms of PSNR, MSE filter orders with different oversample factors is as shown in table1 and graph of PSNR verses filter orders are shown in figure.3. Finally compressed images are applied for image segmentation. Segmentation subdivides an image into its constituent regions or objects. The level to which the subdivision is carried depends on the problem being solved. That is, segmentation should stop when the objects of interest in an application have been solved. We used thresholding segmentation which produce closed, well-defined regions. Because of its intuitive and simplicity of implementation, image thresholding enjoys a central position in application of image segmentation .Two techniques are used for thresholding i.e. global thresholding and local thresholding. In global thresholding select an initial estimate for $\mathrm{T}$ (threshold value). Then segment the image using $\mathrm{T}$. This will produce two groups of pixels:G1 consisting of all pixels with intensity values $\geq T$, and $\mathrm{G} 2$, consisting of pixels with values $<\mathrm{T}$. Compute the average intensity values $\mu 1$ and $\mu 2$ for the pixels in the region G1 and G2. Compute new threshold value $: T=1 / 2(\mu 1+\mu 2)$. In local thresholding technique objective of segmentation is to partition an image into regions. These statistics can characterize the texture of an image because they provide information about the local variability of the intensity values of pixels in an image. For example, in areas with smooth texture, the range of values in the neighborhood around a pixel will be a small value; in areas of rough texture, the range will be larger. Similarly, calculating the standard deviation of pixels in a neighborhood can indicate the degree of variability of pixel values in that region. By using local thresholding segmentation major components of images are exposed. Here results of compressed image of each oversampled filter bank passes through segmentation algorithm, which useful for analysis of image. Here comparison of segmented image of each filter bank is carried out. Figure 9a to 9f shows the resultant of segmented images of FB, which shows that as per as filter order increases for FB, resultant approaches towards near input image.

Table 1 for 15 channel $F B$ with subsample factor 2

\begin{tabular}{|l|l|l|l|l|}
\hline Filter Order & $\begin{array}{l}\text { PSNR in dB } \\
\text { [Cameraman] }\end{array}$ & $\begin{array}{l}\text { PSNR in dB } \\
\text { [man] }\end{array}$ & $\begin{array}{l}\text { PSNR in dB } \\
{[\text { Barba] }}\end{array}$ & $\begin{array}{l}\text { PSNR in dB } \\
\text { [Lena] }\end{array}$ \\
\hline Image format & tif & tif & tif & gif \\
\hline 18 & 42.4086 & 53.6023 & 50.56 & 59.09 \\
\hline 22 & 39.1311 & 40.92 & 43.01 & 48.04 \\
\hline 28 & 37.4989 & 37.61 & 39.08 & 44.79 \\
\hline 32 & 36.8121 & 36.32 & 37.57 & 43.96 \\
\hline 38 & 34.3256 & 32.80 & 34.64 & 39.77 \\
\hline 42 & 33.1565 & 31.35 & 33.09 & 37.64 \\
\hline 60 & 28.29 & 27.47 & 29.17 & 27.77 \\
\hline
\end{tabular}

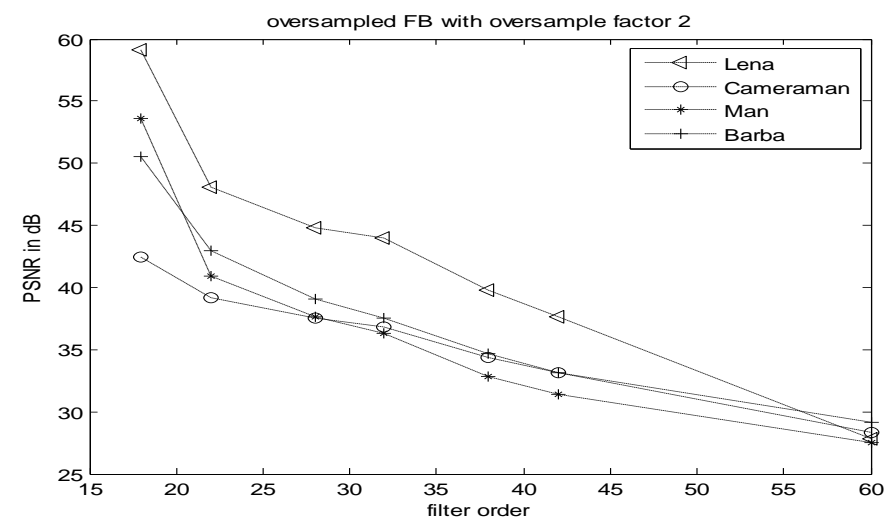

Figure 6 : From table1 graph for oversampled filter bank with oversample factor 2 for images Cameraman ,Barba, Leena, and Man. 


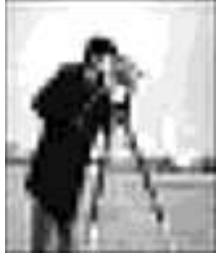

a

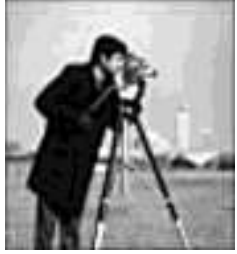

d

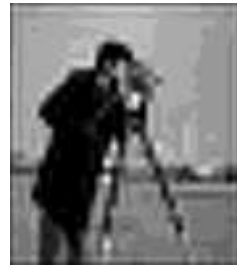

b

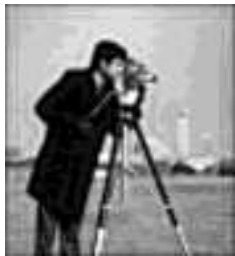

e

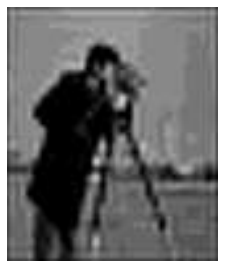

c

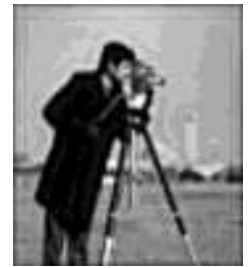

$\mathrm{f}$

Figure 7 :a] Output of Cameraman input FB for filter order=28 and oversampled factor 8 b] Output of FB for filter order $=32$ and oversampled factor $8 \mathrm{c}$ ] Output of $\mathrm{FB}$ for filter order $=38$ and oversampled factor $8 \mathrm{~d}$ ] Output of Cameraman input FB for filter order=28 and oversampled factor 4 e] Output of FB for filter order=32 and oversampled factor $4 \mathrm{f}$ ] Output of FB for filter order $=38$ and oversampled

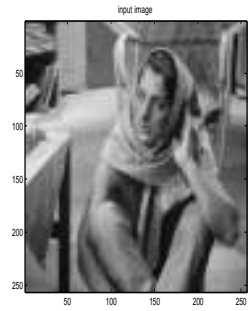

i

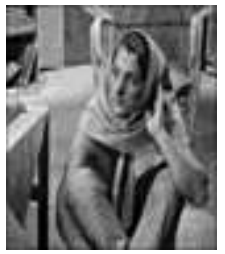

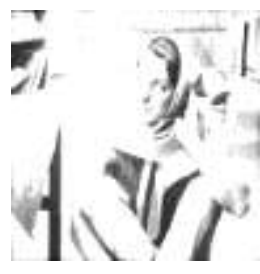

j

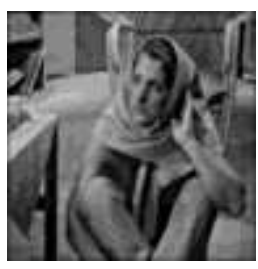

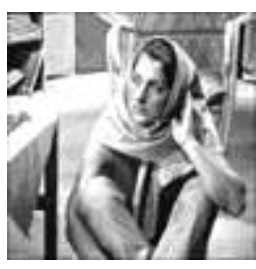

$\mathrm{k}$

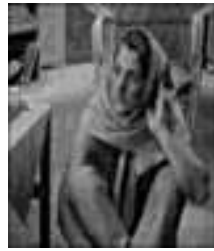

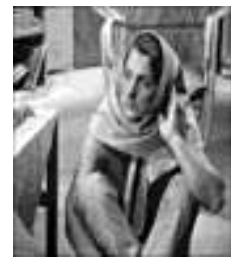

1

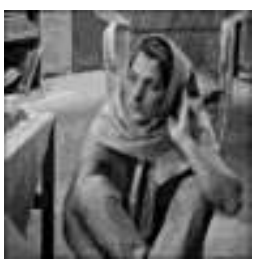

Figure 8 :i] Barba input image j] output of FB for filter order=10 k] output of FB for filter order=18 1] output of FB for filter order $=22 \mathrm{~m}$ ] output of FB for filter order $=28 \mathrm{n}$ ] output of $\mathrm{FB}$ for filter order $=32 \mathrm{o}$ ] output of $\mathrm{FB}$ for filter order $=38 \mathrm{p}$ ] output of FB for filter order $=42$

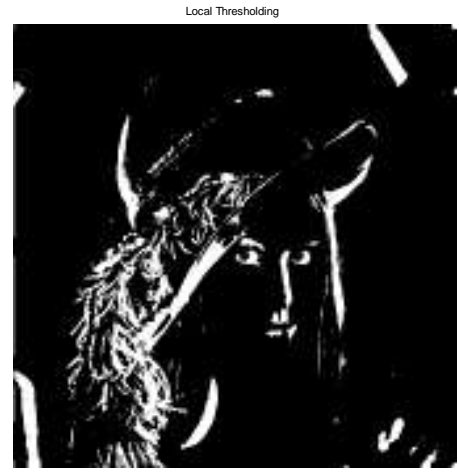

Figure 9a] segmented image of input image 


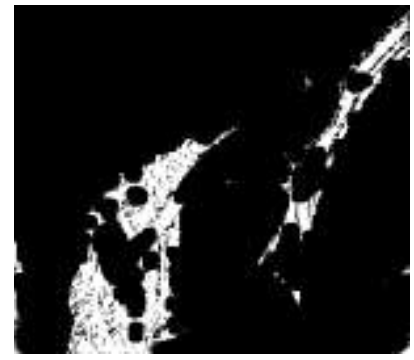

Figure 9b] segmented image of FB with filter order 10

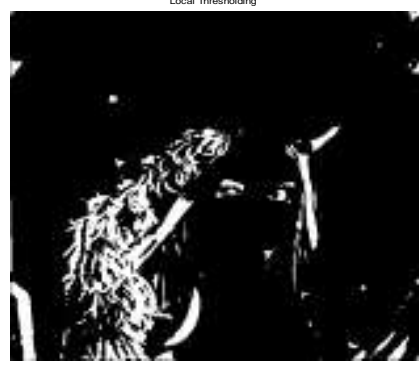

Figure9 c] segmented image of FB with filter order 18

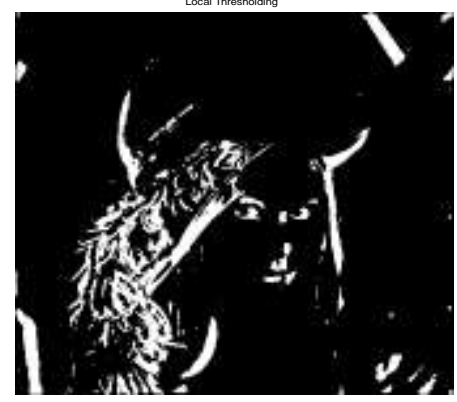

Figure9 d] segmented image of FB with filter order 28

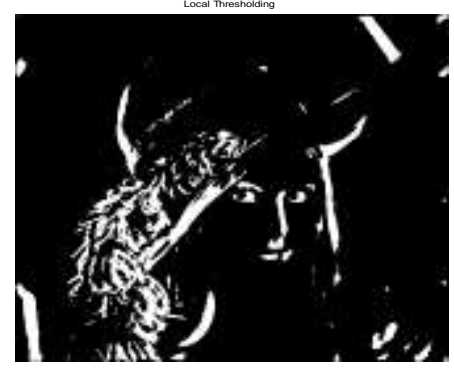

Figure 9 e] segmented image of FB with filter order 38

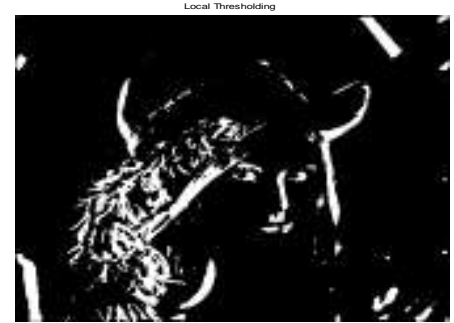

Figure9 f] segmented image of FB with filter order 42 


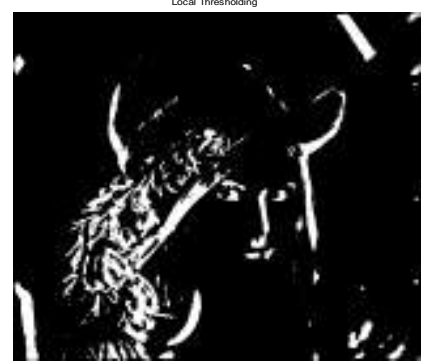

Figure $9 \mathrm{~g}$ ] segmented image of FB with filter order 60

\section{Conclusion:}

In this paper proposed oversampled real valued, even filter order, filter banks have been designed. To investigate oversampled FIR filter bank which gives resultant near perfect reconstruction of input image, different subsampled factors are applied. Subsampled factors 4 and 8 of FIR filter bank are not gives the proper output. Therefore subsample factor 2 is selected to achieve the resultant near input image. Also for design of this FB's, selection of passband frequency range and filter order are most essential to achieve perfect reconstruction output.

\section{References :}

[1] R. Brennan and T. Schneider, "A flexible filterbank structure for extensive signal manipulations in digital hearing aids," in Proc. IEEE International Symposium on Circuits and Systems, May 1998, vol. 6, pp. 569-572.

[2] I. Daubechies, "Orthonormal bases of compactly supported wavelets," Communications on Pure and Applied Mathematics, vol. 41, pp. 909-996, 1988.

[3] S. G. Mallat, "Multifrequency channel decompositions of images and wavelet models," IEEE Transactions on Acoustics,Speech, and Signal Processing, vol. 37, no. 12, pp. 2091-2110,1989.

[4] Hindawi Publishing CorporationEURASIP Journal on Applied Signal Processing Volume 2006, Article ID 42672,Pages 1-16DOI 10.1155/ASP/2006/42672“2D Four-ChannelPerfect Reconstruction Filter Bank”Realized with the 2D Lattice Filter Structure

[5] Moritz Harteneck, Robert W. Stewart and J.M. Paez-Borrallo., A filterbank design for oversampled filter banks without aliasing in the subbands http://citeseer.ist.psu.edu/31230.html

[6] Moritz Harteneck,Stephan Weiss, and Robert W. Stewart ,An oversampled filter bank withdifferent analysis and synthesis filters for the use with adaptive filters http://citeseer.ist.psu.ed

[7] P.P.Vaidyanathan "Multirate systems and filter banks"(.Pearson Education 1993). 